\title{
Satisfacción percibida por los pacientes en el servicio de hemodiálisis del hospital general de La Palma
}

\author{
David Rodríguez González, David Sanjuán Hernández
}

Enfermeros, Servicio de hemodiálisis del Hospital General de la Palma

\section{Resumen:}

En los tiempos actuales en los que la mayoría de los esfuerzos se centran en las nuevas actualizaciones y novedosos tratamientos para los pacientes con Insuficiencia Renal Crónica, nuestro interés, sin embargo, se ha dirigido hacia algo mucho más básico: conseguir saber cómo perciben los pacientes nuestros cuidados y cómo se sienten ellos en el servicio. Por tanto, el objetivo de este estudio es determinar el grado de satisfacción del paciente.

El servicio de Hemodiálisis en la Isla de La Palma lleva desarrollando su actividad desde mayo de 1984, no sólo para atender a los pacientes residentes en la isla, sino también a los transeúntes que, por diversos motivos, se encuentran aquí.

Se ha efectuado un estudio mediante encuestas anónimas y voluntarias efectuadas a pacientes de la Unidad de Hemodiálisis. Hemos utilizado la encuesta SERVQUOS modificada, y la escala Likert.

Se realiza el tratamiento informático de los datos con el programa SPSS-19 para Windows.

Según los resultados obtenidos, el índice neto de satisfacción fue de $86,36 \%$ para el ítem referido a la satisfacción general, teniendo una valoración de excelente. La media de edad de los pacientes fue de 64,63 años; el número medio de ingresos en el últi-

Correspondencia:

David Rodríguez González

Camino Triana, $n^{\circ}$ 176, vivienda 2

38760 Los Llanos de Aridane. Sta Cruz de Tenerife

E-mail: davidhd2@hotmail.com mo año por pacientes fue de $1,08 \%$; el porcentaje de pacientes en lista de trasplante fue del $20,7 \%$.

Los 5 ítems más valorados fueron: la confianza trasmitida, la disposición del personal a prestarle ayuda, el trato personalizado, la amabilidad del personal y la información relacionada con la higiene. Por otro lado, los menos valorados fueron: la señalización para llegar al Servicio, la capacitación del personal, la información médica, la tecnología de los equipos y el tiempo de espera para ser atendido por el médico.

\section{PALABRAS CLAVE:}

- HEMODIÁLISIS

- CALIDAD ASISTENCIAL PERCIBIDA

- CUESTIONARIO SERVQHOS MODIFICADO

\section{Patient-perceived Satisfaction in the Haemo- dialysis Service of La Palma General Hospital}

\section{Abstract}

Although most of the current researches are focussed on new actualizations and innovative treatments, the aim of this study is to find out how patients perceive our health care and how they feel at our Hospital Unit. Thus, the main objective is to find out the degree of patient satisfaction.

Haemodialysis Service in La Palma Island is working since 1984, not only to give treatment to local patients, but also to support the health assistance of outpatients who are temporally on the island because of different reasons. 
Data were collected through anonymous surveys of volunteer patients using the modified SERVQHOS and the Likert scale, too.

Quantitative data analysis was made through SPSS19 program for Windows.

According to the results, the Satisfaction Net Index showed that the $86,36 \%$ of the participants punctuated "excellent" the item referred to the general satisfaction degree. The average age and admissions to hospital during last year were 64.63 years old and $1.08 \%$ respectively. The percentage of patients registered on the kidney transplant waiting list was $20,7 \%$.

The most positively considered features were: The confidence, the staff disposition to help, their kindness and the information related with hygiene. On the other hand, the most negatively viewed items were: the indications to find the dialysis unit, the staff's qualification, medical information, equipment technology and the waiting time to be seen by the doctor.

\section{KEYWORDS:}

- HAEMODIALYSIS

- PATIENT-PERCEIVED QUALITY

- MODIFIED SERVQHOS

\section{Introducción}

La evaluación de la asistencia sanitaria es consustancial a la práctica clínica, las primeras referencias a la misma se remontan a las culturas egipcia o china.

Institucionalmente, el interés por la calidad en la asistencia sanitaria ha crecido en los últimos tiempos tanto en el sistema público como en el sector privado. En este sentido, uno de los pasos más avanzados ha tenido lugar en los servicios de nefrología, y más concretamente en sus unidades de hemodiálisis, tanto centrales como satélites. Sirva de ejemplo, que en los últimos concursos de hemodiálisis en nuestro país, se esté valorando de forma positiva el contar con un sistema de gestión de la calidad certificado, y obligando a los centros a comprometerse en un plazo concreto a establecer un sistema de acreditación de calidad de sus protocolos de actividad $^{1}$.
Un sistema de calidad es un sistema vivo, que tiene en cuenta que las necesidades que se deben satisfacer pueden cambiar con el tiempo, lo que implica una revisión periódica y una adaptación a las nuevas tendencias con introducción de innovaciones cuando sea preciso.

El continuo incremento de la incidencia de enfermedad renal crónica terminal (IRCT), debido a múltiples factores (la mayor longevidad de la población, mayor presencia de patología cardiovascular, especialmente hipertensión vascular, diabetes mellitus), nos ha hecho preguntarnos cómo nuestros pacientes, dentro de sus condiciones individuales, perciben la atención recibida.

En términos generales, se pueden distinguir dos conceptos de calidad: la calidad objetiva y la calidad percibida. La primera se corresponde con la calidad real de los productos, que se puede medir y comparar desde un punto de vista técnico. Sin embargo, muchas veces el consumidor no tiene capacidad para juzgar la calidad objetiva, por lo que es la calidad percibida la que actúa como variable de decisión en la selección de una marca concreta. Según Zeithaml (1988) la calidad percibida es "la opinión del consumidor sobre la superioridad o excelencia de un producto". Dicha opinión se configura, según Cruz y Múgica (1993), a partir de tres tipos de factores:

a) Los atributos intrínsecos del producto o calidad objetiva.

b) Los atributos extrínsecos, como la marca, el envase o la imagen transmitida por la publicidad, a los que el consumidor asocia una determinada calidad.

c) El precio, un atributo extrínseco que debe considerarse separadamente por su especial influencia en la calidad percibida.

- Objetivos:

Objetivo Principal: Determinar el grado de satisfacción del paciente con IRCT en el Servicio de Hemodiálisis del Hospital General de La Palma.

- Objetivos específicos:

a) Definir el perfil del paciente en hemodiálisis en la Isla de La Palma.

b) Detectar áreas de mejora, para establecer estrategias en función de los ítems peor valorados por los pacientes. 
c) Determinar si existen diferencias entre grado de satisfacción de los pacientes y diferente acceso vascular.

d) Determinar valores estadísticos de nuestra unidad como referente de próximos estudios.

\section{Material y métodos}

Nuestra unidad se inauguró en mayo de 1984 y hasta la actualidad da cobertura a los pacientes residentes con IRC en hemodiálisis de la Isla de La Palma, con una población de 87163 habitantes, (INE enero 2011) y además atiende a transeúntes (Tabla I).

\begin{tabular}{|c|c|}
\hline $\begin{array}{l}\text { Ratio enfermera / } \\
\text { paciente }\end{array}$ & 1 enfermera / 4 pacientes \\
\hline $\begin{array}{l}\text { Organización del } \\
\text { trabajo médico }\end{array}$ & $\begin{array}{ll}3 \text { nefrólogas: } & \\
\text { 1) Hospitalización } & 2 \text { turnos (27pac.) } \\
\text { 2) Consulta } & 0 \text { turnos } \\
\text { 3) ITC hospitalización } & 2 \text { turnos (27pac.) }\end{array}$ \\
\hline $\begin{array}{l}\text { Ratio auxiliar / } \\
\text { paciente }\end{array}$ & 1 auxiliar/5-9 pacientes \\
\hline $\begin{array}{l}\text { Formación enfer- } \\
\text { mera }\end{array}$ & $\begin{array}{l}\text { 1-2 meses } \\
\text { Aconsejable curso actualización SEDEN }\end{array}$ \\
\hline Turnos & Mañana/Tarde (4 turnos semanales) \\
\hline Salas & $\begin{array}{l}1 \text { sala agudos/crónicos ( } 18 \text { tomas) } \\
1 \text { sala aislamiento ( } 2 \text { tomas) }\end{array}$ \\
\hline Conexión catéter & Aséptica ( 1 solo enfermero) \\
\hline Catering & Bocadillos, galletas, zumos, yogurt, compotas \\
\hline $\begin{array}{l}\text { Ubicación del } \\
\text { paciente }\end{array}$ & Sillones/Camas \\
\hline $\begin{array}{l}\text { Televisión / } \\
\text { paciente }\end{array}$ & 1 televisor/2 pacientes \\
\hline
\end{tabular}

Tabla I. Datos descriptivos del centro de hemodiálisis.

Se trata de un estudio descriptivo y correlacional, en el cual se han incluido a todos los pacientes con IRC en programa de Hemodiálisis residentes en La Palma, que llevaran más de un mes en programa y que reunieran las condiciones físicas, mentales e idiomáticas necesarias para rellenar el cuestionario $0^{2,3}$.

De los 58 pacientes en tratamiento se han excluido del estudio a 4 pacientes por llevar menos de un mes y una paciente diagnosticada de demencia senil avanzada. Tras informar de los objetivos del estudio, todos los pacientes participantes han dado su consentimiento informado 4 .
Como instrumento de medida hemos usado la encuesta SERVQHOS y se han recogido los datos por medio de un cuestionario anónimo (Anexo I).

La encuesta SERVQHOS es el instrumento que nos permite evaluar la calidad percibida por el paciente, aplicada en estudios de satisfacción de los usuarios de servicios sanitarios, que combina expectativas y percepciones. Se trata de un cuestionario ya validado que ha demostrado una alta consistencia interna, una buena capacidad predictiva, y especialmente una buena estructura factorial, además de discriminar entre pacientes satisfechos e insatisfechos así como, a su vez, permitirnos identificar oportunidades de mejora ${ }^{5,6}$.

Se trata de puntuar 22 ítems. Para facilitar al paciente la realización del cuestionario, hemos agrupado las preguntas en tres apartados temáticos: la unidad de hemodiálisis, el personal de hemodiálisis y el funcionamiento de hemodiálisis.

Cada ítem se puntúa con escala Likert, desde 1 ("mucho peor de lo que esperaba") hasta 5 ("mucho mejor de lo que esperaba").

Hemos modificado el cuestionario para adaptarlo a nuestro estudio y a nuestro Servicio. La información que da la enfermera la hemos dividido en tres ítems:

a) Información relacionada con la higiene corporal.

b) Información relacionada con la alimentación.

c) Información que da la enfermera relacionada con las actividades diarias.

Hemos incluido cuatro preguntas directas ya que éstas se han confirmado en numerosos estudios como criterios predictores de calidad percibida ${ }^{2}$. La primera, sobre el nivel de satisfacción global con el servicio de hemodiálisis; la segunda hace referencia a si el paciente recomendaría el servicio a otras personas; la tercera y cuarta pregunta responden a si el paciente conoce el nombre del médico y si conoce el nombre de la enfermera que le atiende.

La última parte de nuestra encuesta incluye características de los pacientes, divididas en datos socio-demográficos: edad, sexo, estado civil, nivel de estudios, situación laboral y datos clínicos: tiempo en programa de hemodiálisis, acceso vascular, turno de HD, trasplantes. Datos estos, concluyentes para determinar el perfil del paciente ${ }^{7}$. 


\section{Estimado Sr. / Sra.:}

Quienes trabajamos en el Servicio de Hemodiálisis del Hospital General de la Palma estamos interesados en conocer su opinión acerca de la calidad del servicio que se le ha ofrecido durante su estancia en el mismo. Por este motivo nos permitimos dirigirnos a VD. Con el propósito de solicitar su colaboración, con la seguridad de que su opinión y ayuda será de gran utilidad para que podamos hacerlo cada vez mejor. Por supuesto que su colaboración es totalmente voluntaria y completamente anónima. Todo lo que diga será tratado de forma absolutamente confidencial.

Le agradecemos muy sinceramente su interés al realizar la encuesta y su atención para con nuestro trabajo, con la seguridad de que será en beneficio de todos.

Basándose en su experiencia señale si la calidad de la asistencia sanitaria que le ha proporcionado el servicio de hemodiálisis ha sido mejor o peor de lo que Vd. Esperaba. Si cree, por ejemplo, que ha sido MUCHO PEOR de lo que esperaba ponga una X en la casilla 1 . Si ha sido MUCHO MEJOR de lo que esperaba, ponga la $X$ en la casilla 5, y así sucesivamente.

\begin{tabular}{|c|c|c|c|c|c|}
\hline \multicolumn{6}{|c|}{ La calidad de la asistencia sanitaria ha sido } \\
\hline & $\begin{array}{c}\text { Mucho } \\
\text { peor de } \\
\text { lo que } \\
\text { espera- } \\
\text { ba }\end{array}$ & $\begin{array}{c}\text { Peor de } \\
\text { lo que } \\
\text { espera- } \\
\text { ba }\end{array}$ & $\begin{array}{c}\text { Como } \\
\text { se lo } \\
\text { espera- } \\
\text { ba }\end{array}$ & $\begin{array}{c}\text { Mejor } \\
\text { de lo } \\
\text { que es- } \\
\text { peraba }\end{array}$ & $\begin{array}{c}\text { Mucho } \\
\text { mejor } \\
\text { de lo } \\
\text { que es- } \\
\text { peraba }\end{array}$ \\
\hline $\begin{array}{c}\text { Clave } \\
\text { respuesta }\end{array}$ & 1 & 2 & 3 & 4 & 5 \\
\hline
\end{tabular}

\begin{tabular}{|l|l|l|l|l|l|}
\hline En la unidad de Hemodiálisis \\
\hline La facilidad para llegar al hospital ha sido & 1 & 2 & 3 & 4 & 5 \\
\hline $\begin{array}{l}\text { Las indicaciones (señalizaciones) para orien- } \\
\text { tarse hasta llegar al servicio de hemodiálisis }\end{array}$ & 1 & 2 & 3 & 4 & 5 \\
\hline $\begin{array}{l}\text { El estado en que está la sala de hemodiálisis } \\
\text { (apariencia) ha sido }\end{array}$ & 1 & 2 & 3 & 4 & 5 \\
\hline Comodidad de las instalaciones & 1 & 2 & 3 & 4 & 5 \\
\hline $\begin{array}{l}\text { La Tecnología de los equipos médicos para los } \\
\text { diagnósticos y tratamientos ha sido }\end{array}$ & 1 & 2 & 3 & 4 & 5 \\
\hline
\end{tabular}

\begin{tabular}{|l|l|l|l|l|l|}
\hline \multicolumn{2}{|l|}{ El personal de Hemodiálisis } \\
\hline $\begin{array}{l}\text { La apariencia (limpieza y uniforme) del per- } \\
\text { sonal ha sido }\end{array}$ & 1 & 2 & 3 & 4 & 5 \\
\hline $\begin{array}{l}\text { El interés del personal por cumplir lo que } \\
\text { promete ha sido }\end{array}$ & 1 & 2 & 3 & 4 & 5 \\
\hline $\begin{array}{l}\text { La disposición del personal para ayudarle } \\
\text { cuando lo necesita ha sido }\end{array}$ & 1 & 2 & 3 & 4 & 5 \\
\hline $\begin{array}{l}\text { La confianza (seguridad) que el personal } \\
\text { transmite a los pacientes ha sido }\end{array}$ & 1 & 2 & 3 & 4 & 5 \\
\hline $\begin{array}{l}\text { La amabilidad (cortesía) del personal en su } \\
\text { trato con la gente ha sido }\end{array}$ & 1 & 2 & 3 & 4 & 5 \\
\hline $\begin{array}{l}\text { La preparación del personal (capacitación) } \\
\text { para realizar su trabajo ha sido }\end{array}$ & 1 & 2 & 3 & 4 & 5 \\
\hline $\begin{array}{l}\text { El trato personalizado que se da a los pacien- } \\
\text { tes ha sido }\end{array}$ & 1 & 2 & 3 & 4 & 5 \\
\hline $\begin{array}{l}\text { La capacidad del personal para comprender } \\
\text { las necesidades de los pacientes ha sido }\end{array}$ & 1 & 2 & 3 & 4 & 5 \\
\hline $\begin{array}{l}\text { La información que los médicos proporcionan } \\
\text { ha sido }\end{array}$ & 1 & 2 & 3 & 4 & 5 \\
\hline $\begin{array}{l}\text { La información que los médicos dan a los fa- } \\
\text { miliares ha sido }\end{array}$ & 1 & 2 & 3 & 4 & 5 \\
\hline
\end{tabular}

\begin{tabular}{|l|l|l|l|l|l|}
\hline $\begin{array}{l}\text { El interés del personal de enfermería por los } \\
\text { pacientes ha sido }\end{array}$ & 1 & 2 & 3 & 4 & 5 \\
\hline $\begin{array}{l}\text { Información que da la enfermera relacionada } \\
\text { con higiene corporal (catéter/FAV/prótesis) }\end{array}$ & 1 & 2 & 3 & 4 & 5 \\
\hline $\begin{array}{l}\text { Información que da la enfermera relacionada } \\
\text { con alimentación }\end{array}$ & 1 & 2 & 3 & 4 & 5 \\
\hline $\begin{array}{l}\text { Información que da la enfermera relacionada } \\
\text { con las actividades diarias }\end{array}$ & 1 & 2 & 3 & 4 & 5 \\
\hline
\end{tabular}

\begin{tabular}{|l|l|l|l|l|l|}
\hline El funcionamiento de Hemodiálisis \\
\hline $\begin{array}{l}\text { El tiempo de espera para ser atendido por el } \\
\text { médico ha sido }\end{array}$ & 1 & 2 & 3 & 4 & 5 \\
\hline $\begin{array}{l}\text { La rapidez con que consigues lo que se nece- } \\
\text { sita o se pide ha sido }\end{array}$ & 1 & 2 & 3 & 4 & 5 \\
\hline Puntualidad de las sesiones de hemodiálisis & 1 & 2 & 3 & 4 & 5 \\
\hline
\end{tabular}

Marque con una $X$ la casilla que mejor refleje su opinión

\section{Indique su nivel de satisfacción general con los cuidados} sanitarios que ha recibido

\begin{tabular}{|c|c|c|c|}
\hline $\begin{array}{c}\text { Muy } \\
\text { satisfecho }\end{array}$ & $\begin{array}{c}\square \\
\text { Satisfecho }\end{array}$ & $\begin{array}{c}\text { Poco } \\
\text { satisfecho }\end{array}$ & $\begin{array}{c}\text { Nada } \\
\text { satisfecho }\end{array}$ \\
\hline
\end{tabular}

\begin{tabular}{|c|c|c|}
\hline $\begin{array}{c}\square \text { Sin } \\
\text { dudarlo }\end{array}$ & $\square \begin{array}{c}\text { Tengo } \\
\text { dudas }\end{array}$ & $\square$ Nunca \\
\hline
\end{tabular}

Si pudiera elegir, repetiría en el mismo servicio de hemodiálisis

\section{Si pudiera elegir, repetiria en el mismo servicio de hemodiálisis}

\begin{tabular}{|l|c|l|l}
$\square \operatorname{Sin}$ \\
$\begin{array}{c}\text { dudarlo } \\
\text { dudas }\end{array}$ & $\square$ Nunca & $\square$
\end{tabular}

¿Le han realizado en el hospital pruebas o intervenciones sin pedir su permiso?

\begin{tabular}{|c|c|c|c|}
\hline$\square$ & Si & $\square$ & No \\
\hline \multicolumn{4}{|c|}{ ¿A su juicio, ha estado ingresado en el hospital? } \\
\hline $\begin{array}{c}\square \quad \text { Menos } \\
\text { de los } \\
\text { necesario }\end{array}$ & $\begin{array}{l}\square \text { El } \\
\text { tiempo } \\
\text { necesario }\end{array}$ & $\begin{array}{l}\square \quad \text { Más de } \\
\text { lo necesario }\end{array}$ & \\
\hline \multicolumn{4}{|c|}{ ¿Conoce el nombre del médico que le atendía habitualmente? } \\
\hline$\square$ & Si & $\square$ & No \\
\hline
\end{tabular}


¿Conoce el nombre de la enfermera que le atiende habitualmente?

$\square \mathrm{Si}$ $\square \quad$ No
¿Cree que ha recibido suficiente información sobre lo que le pasaba?

\begin{tabular}{|c|c|}
\hline$\square$ Si & $\square$ No \\
\hline
\end{tabular}

Por favor, recuerde contestar a todas las preguntas.

\begin{tabular}{|c|c|c|c|c|}
\hline \multicolumn{3}{|c|}{ Por favor rellene las siguientes casillas } & \multicolumn{2}{|l|}{ Marque con una $x$} \\
\hline \multirow{2}{*}{ Edad___ años } & \multirow{2}{*}{ Indique si es: } & $\square \quad$ Hombre & \multirow{2}{*}{ Estado civil: } & $\square \quad$ Casado/a \\
\hline & & $\square \quad$ Mujer & & $\square$ Soltero/a \\
\hline & & & $\square \quad$ Separado/a & $\square \quad$ Viudo/a \\
\hline \multicolumn{5}{|l|}{ Estudios: } \\
\hline$\square \quad$ Sin Estudios & $\square \quad$ Primarios & $\square \quad$ Bachiller & $\square \quad$ Universitarios & \\
\hline \multicolumn{5}{|l|}{ Actualmente está: } \\
\hline$\square \quad$ Jubilado & $\square \quad$ En paro & $\square \quad$ Trabajando & $\square \quad$ Ama/o de casa & $\square \quad$ Estudiante \\
\hline \multicolumn{5}{|c|}{ Tipo de acceso vascular: } \\
\hline$\square \quad$ FAVI & $\square \quad$ Injerto protésico & $\square \quad$ Catéter Permanente & $\square \quad$ Catéter temporal & \\
\hline
\end{tabular}

¿Qué cree usted que se podría mejorar el servicio de Hemodiálisis?

\section{MUCHAS GRACIAS POR SU COLABORACIÓN}

\section{ANEXO I. Encuesta.}

Para concluir el cuestionario, hemos añadido una última pregunta abierta, en la cual el paciente refiera los aspectos de mejora en el servicio de hemodiálisis.

Se volcaron los datos en una tabla Excel y posteriormente se exportaron al programa de análisis estadístico SPSS-19 para Windows.

Para evaluar el grado de satisfacción, hemos hallado el índice neto de satisfacción (INS) que representa el grado de satisfacción global del paciente y lo hemos obtenido a partir de la pregunta de la encuesta administrada: "Su nivel de satisfacción general con los cuidados sanitarios que ha recibido".

Para realizar el cálculo, se ha tomado la tabla de frecuencia de dicha pregunta (SPSS).
Para distribución de las respuestas se ha utilizado la escala numérico/semántica que definan los valores que consideramos "Calidad" (Tabla II).

$$
\begin{aligned}
& 1 \text { - Muy Satisfecho } \\
& 2 \text { - Satisfecho } \\
& \text { 3 - Poco Satisfecho } \\
& \text { - Nada Satisfecho } \\
& \text { 5 - Sin respuesta }
\end{aligned}
$$

$\mathrm{Y}$ daremos un indicador a cada atributo, quedando de la siguiente forma:

$$
\begin{aligned}
& \text { - } \mathrm{A}=\text { Sin respuesta } \\
& \text { - } \mathrm{B}=\text { Nada Satisfecho } \\
& \text { - } \mathrm{C}=\text { Poco Satisfecho } \\
& \text { - } \mathrm{D}=\text { Satisfecho } \\
& \text { - } \mathrm{E}=\text { Muy Satisfecho }
\end{aligned}
$$


El número de respuestas de cada atributo se calcula con la tabla de frecuencias, quedando como se indica:

- $A=0 ; B=0 ; C=1 ; D=22 ; E=21$

- $N\left(n^{\circ}\right.$ de respuestas significativas $)=A+B+C$ $+\mathrm{D}+\mathrm{E}=44$

Y el cálculo del INS lo realizaremos con la siguiente fórmula:

$$
\text { INS }=\frac{(A \times 0)+(B \times 25)+(C \times 50)+(D \times 75)+(E \times 100)}{N}
$$

Se han entregado un total de 53 cuestionarios y los datos

\begin{tabular}{|c|c|c|c|c|c|}
\hline & \multicolumn{5}{|c|}{ Consideraremos CALIDAD a las puntuaciones con $\mathbf{1}$ y 2 . } \\
\hline & & $\begin{array}{c}\text { Frecuen- } \\
\text { cia }\end{array}$ & $\begin{array}{c}\text { Porcen- } \\
\text { taje }\end{array}$ & $\begin{array}{c}\text { Porcentaje } \\
\text { válido }\end{array}$ & $\begin{array}{l}\text { Porcentaje } \\
\text { acumulado }\end{array}$ \\
\hline \multirow{4}{*}{ 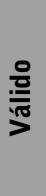 } & $1(E)$ & 21 & 47,7 & 47,7 & 47,7 \\
\hline & $2(D)$ & 22 & 50,0 & 50,0 & 97,7 \\
\hline & $3(\mathrm{C})$ & 1 & 2,3 & 2,3 & 100,0 \\
\hline & Total & 44 & 100,0 & 100,0 & \\
\hline
\end{tabular}
han sido recogidos del 1 de julio al 5 de septiembre del 2012.

Tabla II. Nivel de satisfacción general.

\section{Resultados:}

Los resultados de las características clínicas de los pa-

\begin{tabular}{|c|c|c|c|}
\hline & & Pacientes & $\begin{array}{c}\text { Tanto por } \\
\text { ciento }\end{array}$ \\
\hline \multirow{4}{*}{$\begin{array}{l}\text { Acceso } \\
\text { vascular } \\
\text { actual }\end{array}$} & FAVI & 41 & $77.36 \%$ \\
\hline & Injerto protésico & 2 & $3.77 \%$ \\
\hline & Catéter permanente & 10 & $18.87 \%$ \\
\hline & Catéter temporal & 0 & $0 \%$ \\
\hline \multirow{2}{*}{$\begin{array}{l}\text { Problema } \\
\text { de acceso } \\
\text { vascular }\end{array}$} & Sí & 16 & $30.19 \%$ \\
\hline & No & 37 & $69.81 \%$ \\
\hline \multirow{4}{*}{$\begin{array}{l}\text { Trasplantes } \\
\text { anteriores }\end{array}$} & 0 trasplantes & 50 & $94.34 \%$ \\
\hline & 1 trasplante & 1 & $1.89 \%$ \\
\hline & 2 trasplantes & 1 & $1.89 \%$ \\
\hline & 3 trasplantes & 1 & $1.89 \%$ \\
\hline \multirow{2}{*}{$\begin{array}{l}\text { Lista de } \\
\text { trasplante }\end{array}$} & Sí & 11 & $20.75 \%$ \\
\hline & No & 42 & $79.24 \%$ \\
\hline
\end{tabular}
cientes se especifican en la (tabla III).

Tabla III. Características clínicas de los pacientes.
De los 53 cuestionarios entregados se han recogido 44 . Esto supone una tasa de respuesta del $83 \%$. De los encuestados, el 58,54\% eran hombres, con una media de edad de $63,88 \pm 14,296$ años mientras que las mujeres representan el $41.46 \%$. Su media de edad es del 65,71 $\pm 12,004$ años. El $77.36 \%$ de los encuestados era portador de FAVI; el $3.77 \%$ tenía injerto protésico y el $18.87 \%$ un catéter permanente. El tiempo medio en programa de hemodiálisis fue de 3,59 años \pm 5 años.

Un 66,67\% de los pacientes están jubilados; un 5,13\% está en paro; un $12,82 \%$ trabaja y un $15,38 \%$ se ocupa del hogar.

Un 32,56\% no tiene estudios; un 51,16\% tiene estudios primarios; un $13,95 \%$ estudios secundarios y un $2,32 \%$ estudios universitarios.

No se ha podido hallar correlación estadística entre nivel de satisfacción del paciente y el acceso vascular.

El resultado del INS ha sido de 86,36, siendo los valores de posicionamiento los que se indican: (Tabla IV).

\begin{tabular}{|c|c|}
\hline Valor de NSI & Posicionamiento \\
\hline 85 al00 & Excelente \\
\hline 75 a 84 & Bueno \\
\hline 65 a 74 & Regular \\
\hline 55 a 64 & Malo \\
\hline$<55$ & Muy malo \\
\hline
\end{tabular}

Tabla IV. Valores de posicionamiento INS.

\begin{tabular}{|l|c|c|c|c|c|c|} 
& Edad & $\begin{array}{c}\text { Nivel } \\
\text { Satis- } \\
\text { fac- } \\
\text { ción } \\
\text { Gral. }\end{array}$ & $\begin{array}{c}\text { Infor- } \\
\text { ma- } \\
\text { ción } \\
\text { Higie- } \\
\text { ne }\end{array}$ & $\begin{array}{c}\text { Infor- } \\
\text { mación } \\
\text { Ali- } \\
\text { menta- } \\
\text { ción }\end{array}$ & $\begin{array}{c}\text { Infor- } \\
\text { mación } \\
\text { Activi- } \\
\text { dades } \\
\text { Diarias }\end{array}$ & $\begin{array}{c}\mathbf{N}^{\circ} \\
\text { Ingre- } \\
\text { sos }\end{array}$ \\
\hline Válidos & 41 & 44 & 44 & 44 & 44 & 38 \\
\hline Perdidos & 3 & 0 & 0 & 0 & 0 & 6 \\
\hline Media & 64,63 & 1,55 & 4,61 & 4,48 & 4,27 & 1,08 \\
\hline Mediana & 68,00 & 2,00 & 5,00 & 5,00 & 5,00 & 1,00 \\
\hline Moda & 73 & 2 & 5 & 5 & 5 & 0 \\
\hline
\end{tabular}

Tabla V. Hoja de medias de la calidad percibida.

El $95,45 \%$ de los encuestados recomendaría la unidad de hemodiálisis sin dudarlo, frente al 4,54\%, que tienen dudas. 
Conocen el nombre del médico el $95,35 \%$, mientras que el $4,65 \%$ lo desconoce.

Conocen el nombre de la enfermera el $93,02 \%$ de los pacientes; aunque el $6,98 \%$ responde que no lo conoce.

En la anterior gráfica se especifica la satisfacción percibida referida por los pacientes a las preguntas relacionadas con la información que da la enfermera, teniendo una puntuación de 4,61 sobre 5 la relacionada con la higiene corporal, una puntuación de 4,48 sobre 5 , la relacionada sobre la alimentación y 4,27 sobre 5 en la información sobre las actividades diarias.

El número de ingresos por paciente/año ha sido de 1,08 veces.

En cuanto a los ingresos por pacientes portadores de injerto protésico aumenta hasta llegar al 1,5 de veces al año.

En cuanto a la pregunta abierta "¿Qué cree usted que se podría mejorar en el servicio de hemodiálisis?", del total de sugerencias, el 66,66\% están relacionadas con las pruebas pretrasplante e información sobre la lista de trasplante.

Los ítems más valorados son: la confianza trasmitida, con un $76,74 \%$; la disposición de ayuda, con un $74,42 \%$; el trato personalizado, con un $72,73 \%$; la amabilidad del personal, con un $72,09 \%$ y la información relacionada con la higiene, con un $70,45 \%$. Por otro lado, los menos valorados han sido: la señalización del servicio, con un $56,82 \%$; la capacitación del personal, con un $56,82 \%$; la información médica, con un $55,81 \%$; la tecnología de los equipos, con un $52,27 \%$ y el tiempo de espera con el médico, con un $52,27 \%$.

\section{Discusión}

En la unidad de hemodiálisis del Hospital General de la Palma el perfil del paciente con IRCT es varón, jubilado, con estudios primarios, que utiliza la ambulancia como medio de transporte, dializándose en turnos diurnos (mañana o tarde) mediante FAVI, conocedor del nombre del médico y de la enfermera. La satisfacción percibida por el paciente es excelente y asimismo, recomendaría la unidad sin dudarlo.

Como consecuencia de estas características y debido a múltiples factores - la mayor pluripatología, cronicidad y plurisintomatología $a^{7,8}$, hemos comprobado que el porcentaje de ingresos anuales es de 1,08 veces/año superando a los estudios contrastados de la SEN y oscilando entre valores de 0,47 y 0,57 veces año ${ }^{9}$.

Por esta misma causa, creemos que el porcentaje de pacientes en lista de trasplante en nuestra unidad es inferior a la media, siendo del $20,75 \%$, mientras que en estudios revisados se estima el $22 \%$ (estudios de la SEN) ${ }^{10}$.

En cuanto a los ingresos por pacientes portadores de injerto protésico presentaron cifras de 1,5 veces año, superior a la media del estudio.

En relación al perfil de la unidad de hemodiálisis, nuestro centro realiza 4 turnos de hemodiálisis: mañanas/ tardes, 1 médico /27 pacientes, 1 enfermera /4 pacientes, 1 auxiliar / 5-9 pacientes. Las enfermeras realizan 1-2 meses de prácticas, y se recomienda curso de actualización de SEDEN de Enfermería Nefrológica.

La técnica de punción habitual en FAVI bien desarrollada, es con el bisel hacia abajo. La técnica de conexión aséptica, se realiza por una sola enfermera que prepara el campo estéril.

El catering consiste en: bocadillos, galletas, zumos, yogurt, compota, etc.

Los pacientes son ubicados en sillones o camas y con una ratio de 1 televisor/2 pacientes.

Los aspectos mejor puntuados son: la confianza trasmitida, la disposición de ayuda, el trato personalizado, la amabilidad del personal y la información relacionada con la higiene.

Por otro lado, los aspectos menos puntuados son: la señalización del servicio, la capacitación del personal, la información médica, la tecnología de los equipos y el tiempo de espera para ser atendido por el médico.

\section{Áreas de mejora:}

Con referencia a los aspectos menos puntuados por los pacientes, se podría mejorar su percepción organizando coloquios y charlas dirigidos a ellos y a sus familiares sobre temas como: información general y especifica de su tratamiento IRCT, información sobre la formación requerida a los sanitarios para asistirlos e información sobre los avances tecnológicos y maquinaria que se utiliza en su tratamiento. 
En cuanto a la pregunta abierta sobre qué se podría mejorar en el servicio de HD, sugerimos conferencias y mesas redondas con pacientes trasplantados y con el personal relacionado con la organización nacional de trasplantes a fin de explicarles el proceso para un futuro trasplante.

Dentro de este mismo apartado, nos han manifestado varios pacientes su problemática sobre el dolor producido durante la extracción de la aguja al finalizar el tratamiento. Al no encontrar bibliografía al respecto, podría ser interesante plantear un nuevo estudio sobre este punto.

\section{Agradecimientos}

Agradecemos, en primer lugar, la colaboración de nuestros pacientes porque, sin ellos, este trabajo no hubiera sido posible.

Asimismo queremos agradecer a nuestros compañeros de la unidad, su ayuda, durante el desarrollo del estudio.

Y también al Servicio de Docencia del Hospital General de la Palma, especialmente, al DUE Luis M. Cairos, por su inestimable colaboración.

Recibido: 21 0ctubre 2012

Revisado: 20 Noviembre 2012

Modificado: 6 Mayo 2013

Aceptado: 10 Mayo 2013
3. Hernández Meca ME, Ochando García A, Lorenzo Martínez S, Orbes Cervantes P, y López Revuelta K. Factores determinantes de la satisfacción del paciente en tratamiento renal sustitutivo. Rev Soc Esp Enferm Nefrol 2007; 10 (1); 6-13.

4. García $R$, Palacios $P$, Gallardo Ginés $P$, Rojas Blanco A, Tejuca Marenco A, Tejuca Marenco M. Grado de satisfacción de pacientes con IRC en diálisis hospitalaria vs diálisis domiciliaria. En: Libro de comunicaciones del XXI Congreso Nacional de la Sociedad Española de Enfermería Nefrológica; Salamanca. Barcelona: HOSPAL; 1996.

5. Mira J J, Aranz J, Rodríguez-Marín J, Buil JA, Castell M y Vitaller J. SERVQHOS; un cuestinario para evaluar la calidad percibida de la atención hospitalaria. Medicina Preventiva 1998;IV (4); $4^{a}$ trimestre.

6. Álvarez Benito $M$, Ávila Rodríguez FJ, Barrera Becerra C, Bautista Paloma FJ, Calero Fernández M, Campo García T...et al. Guía de diseño y mejora continua de procesos asistenciales integrados $2^{\mathrm{a}}$ edición. Sevilla; Consejería de salud. 2009.

7. Arenas M. D., Alvárez-Ude F., Egea J. J., Gil M.T., Amoedo M. L., Millán I... et al. Impacto del seguimiento de indicadores de calidad en hemodiálisis. Nefrología. Vol. XXIV. Número 3. 2004.

8. Barranco Martos A, Gómez Salgado J, Peña Amaro P, García Alcaraz F. Tratamiento sustitutivo renal I Hemodiálisis. Cuidados nefrológicos. Fuden 2008. 13-21.

\section{Bibliografía}

1. Barranco Martos A, Peña Amaro P, Gómez Salgado J, García Alcaraz F. Fundamentos de los Cuidados Nefrológicos, Fuden 2008. 297-321

2. Anguera Guinovart $E$, Martínez Bateman F. Estudio multicéntrico sobre la calidad asistencial percibida por los pacientes en programa de hemodiálisis de Tarragona. Rev. Soc. Esp. Enferm. Nefrol.2011;7-9;172-180.
9. Arenas MD, Alvárez-Ude F, Egea JJ, Gil MT, Amoedo ML, Millán I et al. Impacto del seguimiento de indicadores de calidad en hemodiálisis. Nefrología. Vol. XXIV. Número 3. 2004: 265-268.

10. Fernández Fresnedo $G$, de la Oliva Valentín M. Guías SEN Recomendaciones de la Sociedad Española de Nefrología (SEN) y de la organización Nacional de Trasplantes (ONT) sobre trasplante renal de donante vivo. Nefrología. Vol. 30- suplemento 2. 2010.3-13 